\title{
ELECTRICAL SAFETY IN OPERATING ROOMS: REPORT ON REVISIONS TO CSA STANDARD Z 32
}

\author{
J. A. Hopps and H. Callan*
}

CSA STANDARD z 32 was issued in 1963 under the title "Code for Use of Flammable Anaesthetics (Safe Practice for Hospital Operating Rooms)." Its intent was primarily to limit the hazard of ignition or explosion of flammable anaesthetic agents, and secondly, to offer protection against the hazard of electric shock to the patient and to operating room personnel.

At that time it was recognized that electrostatic discharges were capable of igniting explosive gas mixtures. Such discharges were commonplace in Canadian hospital operating rooms, particularly during winter months when central heating systems produced a dry-air environment. Three techniques were employed by the new code to reduce this hazard. First, the code called for a relative humidity of not less than 50 per cent. A specification for operating room floors required conductivity of such an order that electrostatic charges were dissipated while suffcient electrical resistance in the flooring limited the magnitude of shock currents flowing through the body to the grounded floor. The third major specification provided for isolation of the electrical service in the flammable environment so that "grounded" personnel could not receive severe shocks from contact with either power conductor. The code further stipulated that the integrity of the isolated circuit be monitored by a dynamic ground hazard indicator, capable of detecting balanced or unbalanced faults of a resistive, reactive, or combined nature, and designed to alarm when such faults exceeded 1 milliampere ( $\mathrm{rms}$ ).

Implementation of CSA z 32 decreased the incidence of explosion and fire in hospital operating rooms, and the standard has achieved general recognition in Canada. However, a combination of circumstances has necessitated a re-evaluation of the code in recent months, and this has led to major revisions in concept.

In recent years the use of flammable anaesthetic agents has declined greatly. Many hospitals have banned their use in some or all operative areas. At the same time the increasing use of electrical and electronic equipment has accentuated the shock hazard. Moreover, the introduction of conductive probes or electrodes within the body has reduced the threshold level of danger by a factor of 2000 . Ventricular fibrillation, a chaotic heart contraction which destroys effective blood circulation, can result from passage of a $20 \mu \mathrm{A}$ current through the heart muscle. The 1963 code did not provide protection against the internal shock hazard.

The revised standard recognizes this subtle change in the operating room hazard environment. Its title has been changed to "Code for Prevention of Explosions or Electric Shock in Hospital Operating Rooms" to give more emphasis to the shock

"Mr. Hopps is Chairman, Canadian Standards Association Hospital Code Subcommittee on Electronics. Mr. Callan is Chairman, Canadian Standards Association Hospital Code Subcommittee on Electrical Standards. 
hazard. It also provides alternative specifications for a non-flammable environment, for which there has been no safety code up to now.

The code introduces the term Electric Shock Location, defined as a location where special precautions are necessary to protect personnel from electric shock. Operating rooms and postoperative areas are considered to be electric shock locations. Whether or not flammable anaesthetics are used, these areas must comply with the electrical requirements for anaesthetizing locations, as specified in clause 5.4 of the code, with the proviso that in areas where flammable anaesthetics are permanently forbidden, the isolated wiring and equipment need not meet the requirements for class $\mathrm{I}$, division $\mathrm{I}$, group $\mathrm{c}$ locations.

Clause 5.4 in the revised code includes some modifications to the requirements for isolated systems, dynamic ground hazard indicators, signalling, communication, indicator, and low voltage circuits, and additional specifications for the design of defibrillators and stimulators. It also limits the allowable potential gradient between the ground systems of permanent or portable X-ray equipment and of general electrical services to a maximum value of $5 \mathrm{mv}$, rms.

The significant changes in the revised code concern conductive floors. In the flammable environment their requirement continues. For the non-flammable environment, the code recommends non-conductive flooring but adds a caution that footwear which is prone to generation of electrostatic charges - e.g., with rubber, composition, or crepe soles - should be avoided on non-conductive floors, in order to preserve some protection against electrostatic discharge.

The committee in drafting the revisions wished to include a mandatory requirement for a separate grounding conductor in the building service. However, the Canadian Electrical Code still permits use of a conduit ground. Representation will be made to the Part I Committee on this matter, and in the interim the revised operating room code recommends a separate ground wire.

In drafting these revisions, the Committee on Safety Code for Hospital Hazards recognized the need for a non-flammable environment standard. The relaxation of requirements for conductive flooring and explosion-proof circuitry is conditional upon the hospital's assuming responsibility for the maintenance of a non-flammable environment. If a hospital chooses to provide for both flammable and nonflammable environments, they should be physically separated to ensure that no confusion exists, and that flammable agents are not stored in or transported through the non-flammable area. The code requires identification of both areas.

These revisions are intended as an interim measure. They will appear as CSA Standard z 32.1 in May, 1970. A supplementary code, CSA Standard z 32.2, Code for Electric Shock Protection in Hospital Patient Care Areas, is in preparation, and may be available this year. The eventual objective is the complete rewriting of z 32.1, possibly combined with z 32.2 as a general hospital electrical hazard standard. 